\title{
P-Median: A Performance Analysis
}

\author{
María Beatríz Bernábe Loranca ${ }^{1}$, Jorge Ruiz Vanoye ${ }^{2}$, Rogelio González Velázquez ${ }^{1}$, \\ Marco Antonio Rodríguez Flores ${ }^{1}$, Martín Estrada Analco ${ }^{1}$ \\ ${ }^{1}$ Computer Science Department, Benemérita Universidad Autónoma de Puebla, México \\ beatriz.bernabelgmail.com, marco89 rfehotmail.com, \\ rogelio.gzzvzz@gmail.com, mestráda@cs.buap.mx \\ ${ }^{2}$ Universidad Autónoma del Carmen \\ Ciudad del Carmen Campeche, México \\ jorgedruizvanoye.com
}

\begin{abstract}
This work approaches the P-median problem with a partitioning around medoids methodology. This problem has been extensively studied because of the multiple applications and its NP-hard nature; therefore several are the efforts to find optimal solutions.

In this paper we consider two case studies: 1) instances from OR-Library and 2) geographical objects from the metropolitan zone of Toluca, Mexico. The methods we use to work with these data are: Partitioning Around Medoids (PAM), Variable Neighborhood Search (VNS), Simulated Annealing (SA), P-Median with MATLAB (PML) and finally Tabu Search (TS).
\end{abstract}

Keywords: P-median, partitioning, clustering, algorithms.

\section{Introduction}

In territorial partitioning, two models are the most used: the location-allocation and the set partitioning models. These models seek to group small geographical areas called basic units in a given number of bigger geographical clusters, named territories. The territorial partitioning problem can be modeled like a P-median problem with certain restrictions under the concept of partition, this is, if $\Omega=\{x 1, \ldots, x n\}$ is a finite set with $n$ objects we wish to classify and let $k<n$ be the number of classes where we want to group the objects; a partition $P=(C, \ldots, C k)$ from $\Omega$ in $\mathrm{k}$ $\operatorname{ses} C 1, \ldots, C k$, is characterized by the following conditions:

$$
\begin{gathered}
\text { 1. } \Omega=\bigcup_{i=1}^{K} c_{i} \\
\text { 2. } C_{i} \cap C_{j}=\varnothing, \quad \text { for every } i \neq j
\end{gathered}
$$

The P-median problem consists in finding the best configuration of facilities to attend the population's demand in the best way [1]. Given a set of 1000 nodes (coordinates) in the cartesian plane, where every node possesses a certain demand that must 
be fulfilled, 10 service providers must be installed to satisfy this demand at the minimum cost. The cost can be determined in a proportional way to the distance between nodes.

Given the characteristics of this problem, the formulation to solve it was established in [2]. The problem was named $\mathrm{p}$-median. This problem locates $\mathrm{p}$ facilities to minimize the total distance between the demand points and their nearest facilities [1]. For this we solve the allocation problem and minimize the distance and demand of the nodes. The computational complexity of this kind of problems requires using approximate methodologies to give a satisfactory response in regard to quality and time. In this point, two kinds of data have been processed with different heuristic variants based on the P-median model.

\section{K-medoids and P-Median}

The K-medoids and the P-median problems are similar to each other, while the kmedoids problems deals with cluster analysis to form groups or classes of similar objects, the P-median problem is a facility location problem where the goal is to place facilities on a geographical space to satisfy a certain demand in the area while minimizing transport costs and/or other logistical restrictions (like demand values). When K-medoids methodologies like Partitioning Around Medoids (PAM) are applied to group geographical data, they are basically solving a basic P-median problem where the main goal is to minimize distances between objects (similarity) without other logistical restrictions (uncapacitated). In this paper we employ benchmark matrices from OR-Library for uncapacitated p-median problems and real geographical data from the valley of Toluca, Mexico.

In the section below, we present elementary notions about PAM and the P-median problem and the metaheuristics we have employed in our analysis.

\subsection{Partitioning Around Medoids}

In the partitioning around medoids methods, PAM has a good reputation because it's capable to achieve good results. It was developed by Kaufman and Rousseuw [3]. This method assumes that $\mathrm{n}$ objects exist and a representative object is determined for every group to find $\mathrm{K}$-clusters (groups), this representative is a medoid. After selecting the $\mathrm{K}$ medoids, the algorithm tries to analyze the possible pairs of objects, such that every object is grouped with the most similar medoid. The cost is calculated for each combination according to the defined clustering measurement to determine the best configuration that will be used as the starting point for the next iteration. The complexity of a single iteration is $O\left(K(n-K)^{2}\right)$, therefore the computing time is inefficient for high values of $\mathrm{n}$ and $\mathrm{K}$. 


\section{PAM Algorithm}

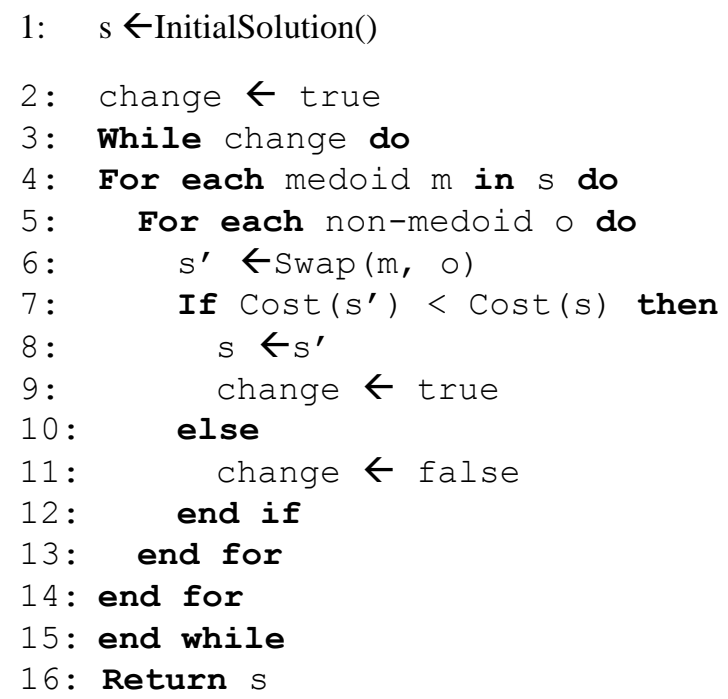

\subsection{P-Median}

The territorial and logistical problems have a wide range of applications; from political districting to social services and commercial territories location, etc. In the related literature, geographical compactness and connectivity criteria are employed to measure the quality of the solutions. According to Kalcsics et al. [4], a territory is geographically compact if its shape is round and isn't distorted; however an exact definition doesn't exist. Generally, the sum of the distances between the basic units (objects) and their representative medoid is the compactness measurement. This is how this problem can be modeled like a P-median one $[5,6]$. The P-median problem can be expressed as follows:

A finite set of objects must be partitioned in exactly p groups. Each of these groups is characterized by one of its objects that was selected to be the median of the group. A distance is specified for every pair of objects and the sum of the distances between objects and their respective medians must be minimized. Its formulation is the following:

Let $N=\{1, \ldots n\}$ be the set of indices for the clients and $J=\{1, \ldots m\}$ the set of indices for the potential locations. Typically $N=J$. For every $(i, j), i$ element of $N, j$ element of $J$. Let $C_{i j}$ the cost of assigning client $i$ to the median located in $j$. The following decision variables are defined:

$$
Y_{j}=\left\{\begin{array}{lr}
1 \text { If the median is located inj } \in J \\
0 \quad \text { otherwise. }
\end{array}\right.
$$




$$
X_{i j}=\left\{\begin{array}{c}
1 \text { If the clienti } \in \text { Nis assigned to the median located in } j \in J . \\
0 \quad \text { otherwise. }
\end{array}\right.
$$

Then, the P-median problem is formulated in the following way:

$$
\operatorname{Min} \sum_{i \in N} \sum_{j \in J} C_{i j} X_{i j}
$$

Subject to:

$$
\begin{aligned}
& \sum_{j \in J} X_{i j}=1 \quad \forall i \in N \\
& \sum_{j \in J} Y_{i j}=p \\
& X_{i j} \leq Y_{j} \quad \forall i \in N, j \in J \\
& X_{i j} \in\{0,1\}, Y_{j} \in\{0,1\} \forall i \in N, j \in J
\end{aligned}
$$

Restrictions (2) ensure that each client is assigned to one single median. Restriction (3) guarantees that exactly p locations for the medians are selected. Restrictions (4) establish that the clients are assigned to a median only if this one has been selected. Finally, the set of restrictions (5) specifies that the decision variables are binary.

The classical version of the P-median problem was formulated in 1960 as an extension to the simple facilities location problems proposed, however an algorithm of polynomial order doesn't exist to solve the P-median problem, this is why is considered an NP-hard problem and is still studied.

We employ three approximation methods to solve P-median and K-medoids. The heuristic methods are commonly used to approximate NP problems. We chose three metaheuristics that have been extensively used to solve the P-median problem in particular [7].

\subsection{Variable Neighborhood Search}

VNS is a metaheuristic technique proposed and described in several works by Mladenovic and Hansen $[8,9,10]$. The basic idea is to combine the application of a local search procedure with a systematic search neighborhood change. The algorithm applies the local search to a solution from the neighborhood of the best solution stored (current solution). If it's impossible to improve this current solution, a bigger neighborhood is considered. When a better solution is obtained the process is restarted. It tries to exploit the idea that the local optimums tend to gather only in certain regions. A more recent tutorial can be found in [11].

The following algorithm shows how the solutions are obtained [12].

\section{VNS Algorithm.}

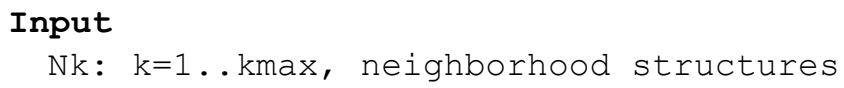




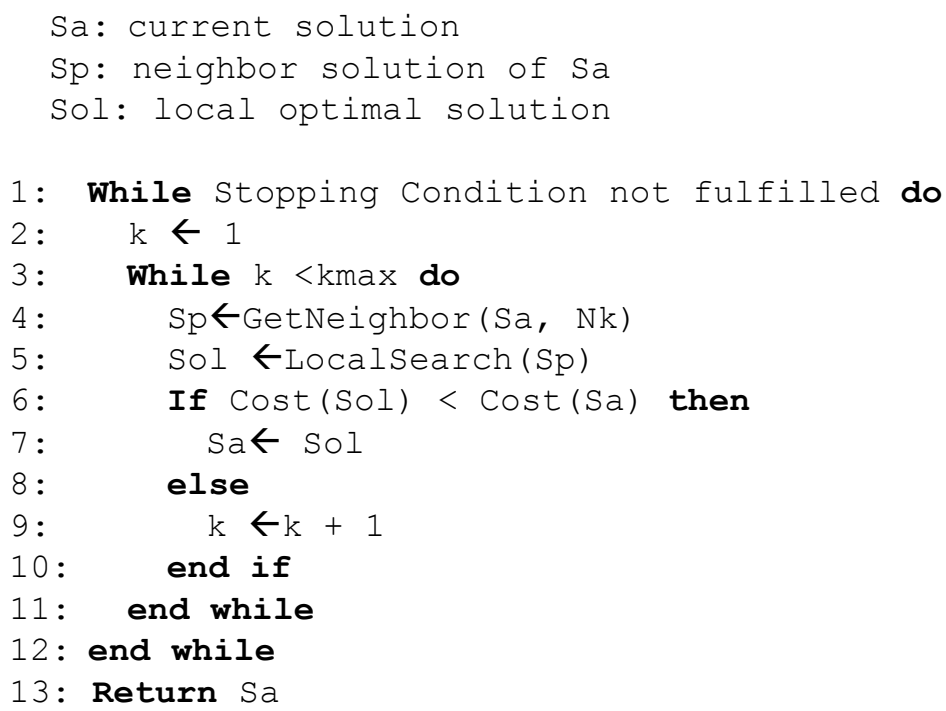

\subsection{Simulated Annealing}

Simulated Annealing is a neighborhood search algorithm with a probability of acceptance criterion based on thermodynamics. It's an optimization method inspired on the metal quenching process used around the 500's B.C. The metal quenching process consists of three phases: a warming phase up to a certain temperature; during the second phase the high temperature is maintained, this allows the molecules to be arranged into minimum energy states, followed by a controlled cooling phase to increase the size of its crystals and reduce its defects. The Metropolis algorithm proposed in 1953 [13] is the pioneer of the simulated annealing methods but Kirkpatrick and Gelatt were the first ones to apply it to optimization problems to find solutions for the travelling salesman problem with several cities [14].

\section{SA Algorithm}

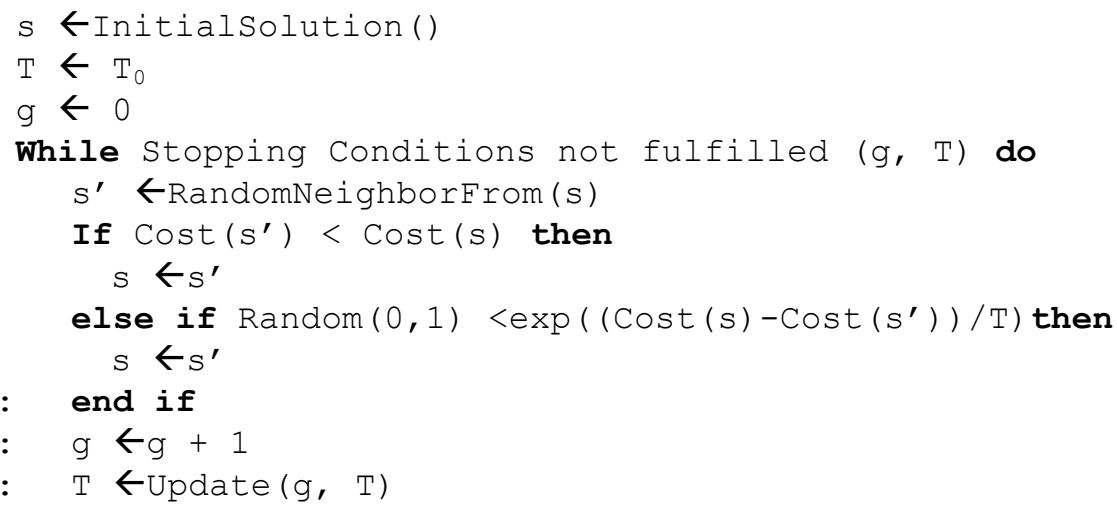


María Beatríz Bernábe Loranca, Jorge Ruiz Vanoye, Rogelio González Velázquez, et al.

\section{3: end while \\ 14: Return s}

\subsection{Tabu Search}

Tabu Search emerged from diverse works published in the late 70's. Despite the fact that the main concepts and strategies behind it already existed, it was in 1989 when the name and methodology were formally established by Fred Glover and Manuel Laguna in the homonymous book Tabu Search [15].

TS is a metaheuristic that guides one or more local heuristics to reach zones of the solution space that regular heuristics usually miss. The local heuristic procedure is an operation used to move between solutions within a defined neighborhood, until reaching a local optimum or fulfilling a stopping criterion. A component from TS that distinguishes it from other metaheuristics is the use of adaptive memory that allows a more flexible search behavior because it stores relevant data about the search process, for example, attributes of the current solution to avoid exploring the same zones of the solution space more than once or to guide the search towards unexplored zones. In this way the motor behind TS is an intelligent and strategic guide capable of making decisions instead of relying on randomness and probability like simulated annealing. This is the biggest contrast between TS and most metaheuristics.

\section{TS Algorithm.}

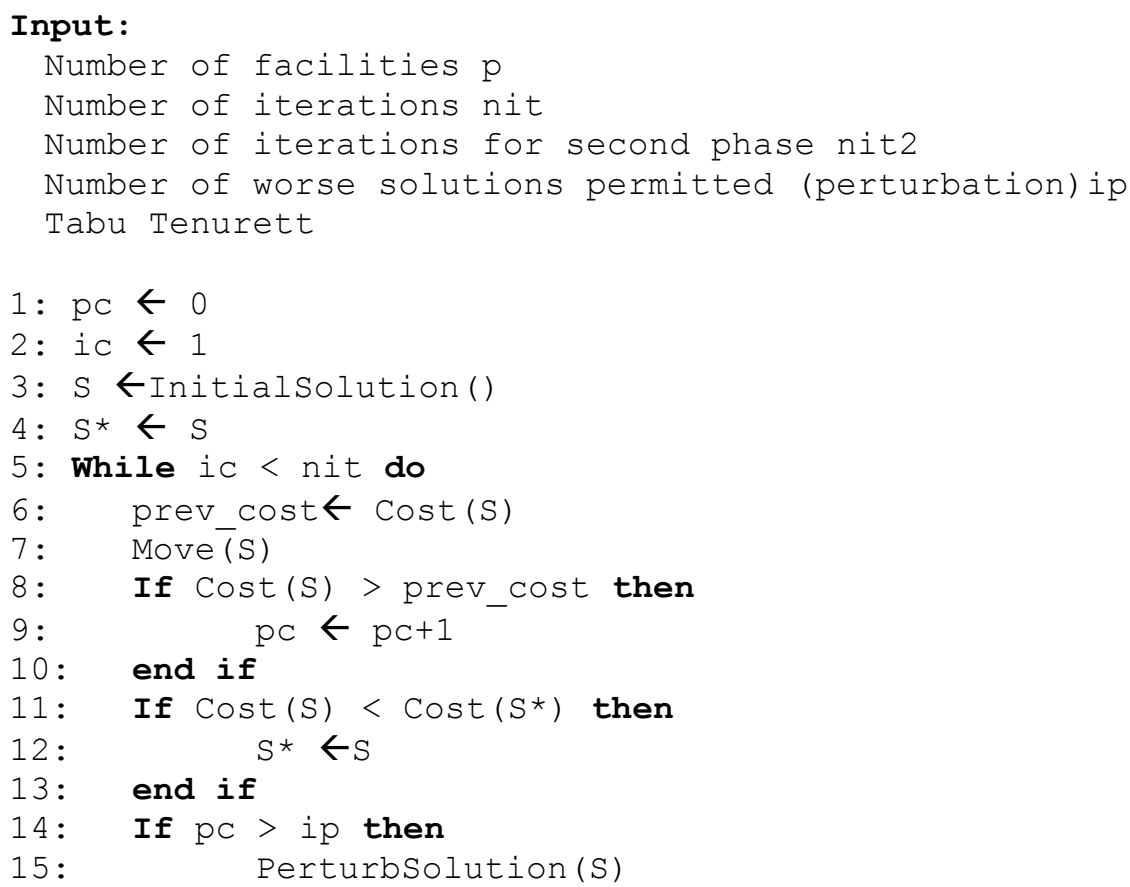




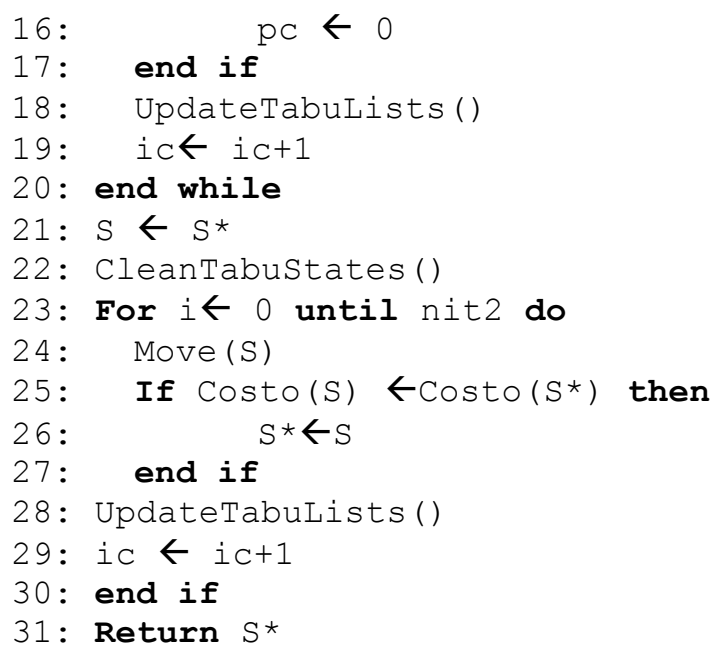

The initial solution is generated with the Stingy Drop method, which has given great results to generate initial solutions $[7,16]$.

Whereas the Move function is a modified faster version of the interchange or swap method proposed by Withtaker that evaluates the profit of interchanging a facility with a candidate facility, in our case we randomly select a cluster and its objects are evaluated as potential facilities and the most profitable is swapped with the medoid [17].

\section{Experimentation}

The experiments made were tested in a Samsung RV415 laptop with 2GB of RAM and $\mathrm{AMD} \AA \mathrm{E}-350 \mathrm{CPU}$ at $1.60 \mathrm{GHz}$.

The instances we present bellow were evaluated with VNS, PAM, SA, PML (MATLAB) and TS. The first instances are available in [18] and are widely used in literature to test P-median algorithms. The second instances belong to a real map of the Toluca valley obtained from the National Institute of Statistics, Geography and Informatics of Mexico (INEGI). We present our results in tables 1, 2 and 3 in section 4.

\subsection{OR-Library - Uncapacitated P-Median}

OR-Library is a collection of test data sets for a variety of Operations Research (OR) problems. OR-Library was originally described in J.E.Beasley [19]. We have taken the 40 available uncapacitated p-median data files that range from 100 to 900 nodes. See Table 1 and 2. 


\subsection{The Valley of Toluca}

This map represents the basic geostatistical areas defined by a census from the year 2000 by the INEGI. It contains 469 objects and our results can be seen in table 3 .

\section{Results}

In this section we cover the results we obtained in several test runs proposed for our data sets.

\subsection{OR-Library Instances}

In tables 1 and 2, we see the results for the OR-Library instances. We can observe that PAM and TS (table 2) attain the best results in comparison with VNS, SA and PML (Matlab), however PML has a clear advantage in regard to computing time.

Table 1. Uncapacitated P-median problems from OR-Library (Worst methods)

\begin{tabular}{|c|c|c|c|c|c|c|c|}
\hline \multirow[t]{2}{*}{ Pmed } & \multicolumn{3}{|c|}{ OR-Library } & \multicolumn{2}{|l|}{ VNS } & \multicolumn{2}{|l|}{ SA } \\
\hline & Nodes & $\mathrm{P}$ & Best & Cost & Time (sec) & Cost & Time (sec) \\
\hline 1 & 100 & 5 & 5819 & 5819 & 103 & 6209 & 28 \\
\hline 2 & 100 & 10 & 4093 & 4341 & 180 & 4646 & 70 \\
\hline 3 & 100 & 10 & 4250 & 4467 & 180 & 4785 & 47 \\
\hline 4 & 100 & 20 & 3034 & 3380 & 250 & 3693 & 93 \\
\hline 5 & 100 & 33 & 1355 & 1664 & 360 & 1820 & 119 \\
\hline 6 & 200 & 5 & 7824 & 7917 & 330 & 8349 & 49 \\
\hline 7 & 200 & 10 & 5631 & 5952 & 540 & 6446 & 104 \\
\hline 8 & 200 & 20 & 4445 & 5204 & 1003 & 5536 & 174 \\
\hline 9 & 200 & 40 & 2734 & 3385 & 1860 & 3626 & 286 \\
\hline 10 & 200 & 67 & 1255 & 1700 & 1980 & 1850 & 907 \\
\hline 11 & 300 & 5 & 7696 & 7803 & 720 & 8346 & 149 \\
\hline 12 & 300 & 10 & 6634 & 7200 & 900 & 7717 & 298 \\
\hline 13 & 300 & 30 & 4374 & 5126 & 1860 & 5475 & 692 \\
\hline 14 & 300 & 60 & 2968 & 3823 & 1440 & 3992 & 71 \\
\hline 15 & 300 & 100 & 1729 & 2464 & 240 & 2558 & 1721 \\
\hline 16 & 400 & 5 & 8162 & 8423 & 300 & 8958 & 220 \\
\hline 17 & 400 & 10 & 6999 & 7651 & 540 & 8197 & 322 \\
\hline 18 & 400 & 40 & 4809 & 5821 & 2700 & 6038 & 505 \\
\hline 19 & 400 & 80 & 2845 & 3747 & 2760 & 3881 & 2036 \\
\hline 20 & 400 & 133 & 1789 & 2647 & 2040 & 2755 & 1909 \\
\hline 21 & 500 & 5 & 9138 & 9557 & 240 & 10231 & 102 \\
\hline 22 & 500 & 10 & 8579 & 9433 & 300 & 9802 & 170 \\
\hline 23 & 500 & 50 & 4619 & 5645 & 3600 & 5941 & 839 \\
\hline 24 & 500 & 100 & 2961 & 3974 & 600 & 4065 & 1440 \\
\hline 25 & 500 & 167 & 1828 & 2726 & 720 & 2852 & 240 \\
\hline 26 & 600 & 5 & 9917 & 10312 & 60 & 10869 & 14 \\
\hline
\end{tabular}


P-Median: A Performance Analysis

\begin{tabular}{|l|l|l|l|l|l|l|l|}
\hline 27 & 600 & 10 & 8307 & 9065 & 120 & 9511 & 25 \\
\hline 28 & 600 & 60 & 4498 & 5664 & 480 & 5799 & 141 \\
\hline 29 & 600 & 120 & 3033 & 4114 & 780 & 4176 & 70 \\
\hline 30 & 600 & 200 & 1989 & 2960 & 1320 & 3058 & 47 \\
\hline 31 & 700 & 5 & 10086 & 10528 & 70 & 11157 & 93 \\
\hline 32 & 700 & 10 & 9297 & 10383 & 120 & 10818 & 119 \\
\hline 33 & 700 & 70 & 4700 & 6007 & 720 & 6166 & 49 \\
\hline 34 & 700 & 140 & 3013 & 4193 & 1500 & 4286 & 104 \\
\hline 35 & 800 & 5 & 10400 & 11037 & 120 & 11698 & 174 \\
\hline 36 & 800 & 10 & 9934 & 9994 & 180 & 11544 & 250 \\
\hline 37 & 800 & 80 & 5057 & 6460 & 1620 & 6715 & 50 \\
\hline 38 & 900 & 5 & 11060 & 11725 & 180 & 12252 & 10 \\
\hline 39 & 900 & 10 & 9423 & 10570 & 300 & 11017 & 25 \\
\hline 40 & 900 & 90 & 5128 & 6632 & 2460 & 6803 & 40 \\
\hline
\end{tabular}

Table 2. Uncapacitated P-median problems from OR-Library (Best methods)

\begin{tabular}{|c|c|c|c|c|c|c|c|}
\hline \multirow[t]{2}{*}{ pmed } & \multirow{2}{*}{$\begin{array}{c}\text { OR-Lib } \\
\text { Best }\end{array}$} & \multicolumn{2}{|c|}{ PAM } & \multicolumn{2}{|c|}{ PML (Matlab) } & \multicolumn{2}{|c|}{ TS } \\
\hline & & Cost & Time (sec) & Cost & Time (sec) & Cost & Time (sec) \\
\hline 1 & 5819 & 5819 & 0 & 5891 & 0.039791 & 5819 & 2.556 \\
\hline 2 & 4093 & 4105 & 0 & 4118 & 0.049311 & 4093 & 1.672 \\
\hline 3 & 4250 & 4250 & 0 & 4399 & 0.056247 & 4250 & 1.604 \\
\hline 4 & 3034 & 3046 & 1 & 3088 & 0.083438 & 3041 & 5.703 \\
\hline 5 & 1355 & 1355 & 1 & 1378 & 0.13098 & 1394 & 5.928 \\
\hline 6 & 7824 & 7824 & 0 & 8027 & 0.075893 & 7824 & 49.28 \\
\hline 7 & 5631 & 5645 & 1 & 5646 & 0.14841 & 5631 & 21.744 \\
\hline 8 & 4445 & 4457 & 2 & 4472 & 0.25138 & 4451 & 19.764 \\
\hline 9 & 2734 & 2753 & 8 & 2841 & 0.49444 & 2804 & 31.729 \\
\hline 10 & 1255 & 1263 & 14 & 1295 & 0.83192 & 1318 & 25.288 \\
\hline 11 & 7696 & 7696 & 0 & 7721 & 0.15035 & 7696 & 145.137 \\
\hline 12 & 6634 & 6634 & 1 & 6651 & 0.28253 & 6634 & 63.67 \\
\hline 13 & 4374 & 4374 & 20 & 4467 & 0.81831 & 4388 & 48.169 \\
\hline 14 & 2968 & 2974 & 56 & 3013 & 1.5988 & 3091 & 37.845 \\
\hline 15 & 1729 & 1738 & 82 & 1761 & 2.6418 & 1858 & 48.857 \\
\hline 16 & 8162 & 8162 & 1 & 8232 & 0.27005 & 8162 & 222.629 \\
\hline 17 & 6999 & 6999 & 2 & 7019 & 0.47927 & 6999 & 97.449 \\
\hline 18 & 4809 & 4811 & 67 & 4873 & 1.8845 & 4840 & 25.538 \\
\hline 19 & 2845 & 2859 & 296 & 2899 & 3.6658 & 2927 & 29.422 \\
\hline 20 & 1789 & 1805 & 600 & 1866 & 6.0295 & 1882 & 36.45 \\
\hline 21 & 9138 & 9138 & 0 & 9138 & 0.38812 & 9138 & 164.141 \\
\hline 22 & 8579 & 8669 & 4 & 8670 & $0-74461$ & 8579 & 58.606 \\
\hline 23 & 4619 & 4619 & 160 & 4694 & 3.5808 & 4664 & 58.606 \\
\hline 24 & 2961 & 2965 & 938 & 3009 & 7.1164 & 3093 & 127.046 \\
\hline 25 & 1828 & 1844 & 1608 & 1896 & 11.9582 & 1937 & 132.722 \\
\hline 26 & 9917 & 9917 & 2 & 10093 & 0.56943 & 9917 & 389.316 \\
\hline 27 & 8307 & 8307 & 9 & 8364 & 1.0522 & 8307 & 68.365 \\
\hline 28 & 4498 & 4515 & 605 & 4579 & 6.207 & 4551 & 35.594 \\
\hline 29 & 3033 & 3039 & 2101 & 3104 & 12.3188 & 3181 & 66.12 \\
\hline
\end{tabular}


María Beatríz Bernábe Loranca, Jorge Ruiz Vanoye, Rogelio González Velázquez, et al.

\begin{tabular}{|c|c|c|c|c|c|c|c|}
\hline 30 & 1989 & 2009 & 2208 & 2037 & 20.3498 & 2119 & 105.318 \\
\hline 31 & 10086 & 10086 & 2 & 10086 & 0.74536 & 10086 & 479.083 \\
\hline 32 & 9297 & 9301 & 8 & 9331 & 1.429 & 9310 & 109.158 \\
\hline 33 & 4700 & 4703 & 1495 & 4798 & 9.7708 & 4735 & 47.558 \\
\hline 34 & 3013 & 3026 & 4685 & 3097 & 19.7808 & 3168 & 119.309 \\
\hline 35 & 10400 & 10400 & 2 & 10406 & 0.96892 & 10400 & 413.429 \\
\hline 36 & 9934 & 9934 & 10 & 9954 & 1.8497 & 9934 & 141.098 \\
\hline 37 & 5057 & 5064 & 2092 & 5118 & 14.4414 & 5278 & 68.316 \\
\hline 38 & 11060 & 11060 & 8 & 11153 & 1.2062 & 11060 & 86.544 \\
\hline 39 & 9423 & 9423 & 13 & 9451 & 2.3816 & 9423 & 99.102 \\
\hline 40 & 5128 & 5138 & 5076 & 5190 & 20.838 & 5214 & 76.852 \\
\hline
\end{tabular}

\subsection{Toluca Valley}

In table 3, we present our results obtained for 15 instances using our second data set. We can see that PAM and TS obtain the best results compared with the rest of our algorithms. However because of its intensification, PAM requires a considerable time, for example when P equals 200 PAM finds a solution in 25200 seconds, PML does it in 14.7711 seconds and TS, 34.504.

With this map TS achieves good computing times and the solutions are better than PML, except for instances 10,11 and 12 and surpasses PAM in tests 2 and 4. After these, VNS is the next method with good results and at last SA. The nomenclature for table 3 is $n$ : test number, P: medians (number of groups), T: computing time in seconds and $\mathrm{C}$ : the cost attained.

Table 3. Toluca Valley (460 nodes)

\begin{tabular}{|l|l|l|l|l|l|l|l|l|l|l|l|}
\hline $\mathrm{n}$ & $\mathrm{P}$ & $\mathrm{VNS}$ & $\mathrm{PAM}$ & \multicolumn{3}{l|}{ SA } & \multicolumn{3}{l|}{ PML (Matlab) } & \multicolumn{2}{l|}{ TS } \\
\cline { 3 - 13 } & & $\mathrm{C}$ & $\mathrm{T}$ & $\mathrm{C}$ & $\mathrm{T}$ & $\mathrm{C}$ & $\mathrm{T}$ & Cost & $\mathrm{T}$ & $\mathrm{C}$ & $\mathrm{T}$ \\
\hline 1 & 5 & 24.24951 & 14 & 23.9643 & 7 & 25.0138 & 0 & 24.3004 & 0.37133 & 23.9643 & 12.767 \\
\hline 2 & 10 & 16.8784 & 21 & 15.837 & 17 & 17.5422 & 1 & 16.8496 & 0.97753 & 15.5434 & 8.594 \\
\hline 3 & 15 & 13.8743 & 28 & 12.3637 & 56 & 14.74491 & 1 & 13.4858 & 1.1002 & 12.3767 & 15.238 \\
\hline 4 & 20 & 12.0909 & 46 & 10.4553 & 120 & 12.6997 & 2 & 11.2325 & 1.4222 & 10.3671 & 12.949 \\
\hline 5 & 30 & 9.682202 & 60 & 8.0539 & 300 & 10.2202 & 2 & 8.5286 & 2.1689 & 8.0743 & 11.361 \\
\hline 6 & 33 & 9.416298 & 53 & 7.496998 & 420 & 9.552296 & 2 & 7.9745 & 2.4193 & 7.5694 & 11.887 \\
\hline 7 & 40 & 8.322398 & 61 & 6.435499 & 660 & 8.834101 & 3 & 6.8658 & 2.9317 & 6.5874 & 10.973 \\
\hline 8 & 67 & 5.985501 & 62 & 4.360599 & 2880 & 6.3118 & 5 & 4.6986 & 5.8809 & 4.5531 & 11.652 \\
\hline 9 & 80 & 5.3999 & 108 & 3.711501 & 2880 & 5.554698 & 5 & 4.0329 & 8.1257 & 3.9411 & 12.432 \\
\hline 10 & 90 & 4.7458 & 120 & 3.3553 & 3600 & 5.7672 & 5 & 3.2601 & 9.8468 & 3.5681 & 14.222 \\
\hline 11 & 100 & 4.494802 & 182 & 3.0419 & 3603 & 4.561401 & 5 & 2.7308 & 14.0718 & 3.2501 & 13.954 \\
\hline 12 & 133 & 3.419801 & 241 & 2.3111 & 11820 & 3.6658 & 6 & 2.4554 & 13.7786 & 2.4568 & 15.705 \\
\hline 13 & 140 & 3.360899 & 256 & 2.1862 & 12000 & 3.4056 & 6 & 2.3286 & 9.5345 & 2.3209 & 25.646 \\
\hline 14 & 150 & 3.113098 & 257 & 2.0272 & 18000 & 3.1526 & 6 & 2.1564 & 10.1451 & 2.1443 & 28.511 \\
\hline 15 & 200 & 2.221599 & 579 & 1.4159 & 25200 & 2.2481 & 8 & 1.5033 & 14.7711 & 1.4999 & 34.504 \\
\hline
\end{tabular}




\section{Conclusions}

Our analysis of the PAM, VNS, SA, PML and TS algorithms allows us to see the strategies that achieve the best results.

From tables 1 and 2, we clearly see that PAM is the one that obtains the most optimal solutions but its computing time is excessive for instances with several nodes and high values of P. In table 3, for example, we see that PAM takes 25200 seconds to return a solution with 469 nodes and $200 \mathrm{P}$. In this matter, is clear that PML (MATLAB) works faster by returning a solution in less than 15 seconds for the biggest instance.

On the other hand, TS reaches the best known solutions for the OR-Library instances in most of the cases. PML gives good results for certain instances (for example test run 31 in 0.74536 seconds) but the cost of the solutions is inferior to the costs achieved by TS in most instances and the computing time for TS is a clear improvement from PAM. This can be observed in table 2, however the computing time for TS can be dramatically reduced according to the number of iterations, for example, test 40 finished in 76 seconds while instance 35, which is smaller, in 413 seconds.

VNS and SA present the same issue, their computing times rely on the parameters passed to the algorithms and could respond in much shorter times with the appropriate values, however for these two we have used first improve strategies (the first improving move is selected). This strategies sacrifice optimality for speed, therefore despite our attempt to give them more time to reach a solution (high parameters) they have given the worst results in both tests, this tells us that completely random strategies are unreliable even with extended execution times.

With TS we have implemented best improve strategies (the best move is chosen) while maintaining a good performance, for example for the smaller instances of ORLibrary (100 to 300 nodes) the algorithm handles 40000 to 70000 iterations in less than 5 seconds.

PAM relies on a thorough search until no improvement is made having a big influence on the speed to find a solution for big values of $\mathrm{P}$ and more than 400 nodes. This makes it the best choice only for small problems to find optimal solutions in competitive times.

In table 3, the map of Toluca, a small sized problem TS is the best choice in terms of time versus optimality because it surpasses the cost of the solutions obtained by PML in several occasions under 35 seconds and in tests 2 and 4 finds better solutions than PAM.

In conclusion the best improve strategies are the most adequate to reach optimal solutions, however a balance should exist between random and intensification strategies to reduce the effect on the performance like we did it in our TS algorithm. Our current challenge is to improve this algorithm to reach the best known solutions for the hardest p-median problems from OR-Library while maintaining a good performance.

\section{References}


1. Daskin, M. S.: Network and Discrete Location: Models, Algorithms and Applications, John Wiley and Sons, Inc., New York (1995)

2. Hakimi, S.: Optimum location of switching centers and the absolute centers and medians of a graph. Operations Research, 12, 450-459 (1964)

3. Kaufman, L., Rousseeuw, P.: Clustering by Means of Medoids. In: Y. Dodge, Editor, Statistical Data Analysis Based on the L1-Norm and Related Methods, Amsterdam: NorthHolland, 405-416 (1987)

4. Kalcsics, J., Nickel, S., Schröder, M.: Towards a Unified Territory Design Approach: Applications, Algorithms and GIS Integration. TOP, 13, 1-74 (2005)

5. Hess, S.W., Samuels, S.A.: Experiences with a sales districting model: criteria and implementation. Management Science, 18, 41- 54 (1971)

6. Zoltners, A., Sinha, P.: Sales territory design: thirty years of modeling and implementation. Marketing Science 24, 313-331 (2005)

7. Mladenovic, N., Brimberg, J., Hansen, P., Moreno, J. A.: The p-median problem: A survey of metaheuristic approaches. European J Operational Research, 179 (2007)

8. Hansen P., Mladenovic, N.: Variable neighborhood search, Les Cahiers du GERAD 96-49 (1996)

9. Mladenovic, N., Hansen, P.: Variable Neighborhood Search. Computers \& Operations Research. 24, 1097-1100 (1997)

10. Hansen, P., Mladenovic N., Pérez M. J.: Variable Neighbourhood Search. Inteligencia Artificial, Revista Iberoamericana de Inteligencia Artificial. No.19 ISSN 1137-3601, 77-92 (2003)

11. Hansen, P., Mladenovic, N.: Variable Neighborhood Search. In Fred Glover \& Gary. A. Kochenberger (Eds.), Handbook of Metaheuristics, Kluwer Academic Publishers, 145-170 (2003)

12. Pelta, A.D.: Algoritmos heurísticos en bioinformática. PhD dissertation, Universidad de Granada, España (2000)

13. Metropolis, N., Rosenbluth, A., Teller, E.: Equation of state Calculations by Fast Computing Machines, J. Chem. Phys., 21, 6, 1087-1092 (1953)

14. Kirkpatrick, S., Gelatt, C. D., Vecchi, M. P.: Optimization by Simulated Annealing. Science, 220, 671-680 (1983)

15. Glover, F., Laguna, M.: Tabu Search. Kluwer Academic Publishers, Norwell, MA, USA (1997)

16. Al-khedhairi, A.: Simulated Annealing Metaheuristic for Solving P-Median Problem. Int. J. Contemp. Math. Sciences, 3, 1357-1365 (2008)

17. Resende, M., Werneck, R.: A fast swap-based local search procedure for location problems. Annals of Operational Research, 150, 205-230 (2007).

18. OR-library http://people.brunel.ac.uk/ mastjjb/jeb/orlib/pmedinfo.html Retrieved on August 252014

19. OR-Library: distributing test problems by electronic mail. Journal of the Operational Research Society, 41, 1069-1072 (1990) 